\title{
In situ characterization of AA1050 recrystallization kinetics using high temperature nanoindentation testing
}

\author{
Paul Baral ${ }^{1}$, Mathilde Laurent-Brocq ${ }^{2}$, Gaylord Guillonneau ${ }^{1}$, \\ Jean-Michel Bergheau ${ }^{3}$, Jean-Luc Loubet ${ }^{1}$, Guillaume Kermouche ${ }^{4}$
}

${ }^{1}$ Université de Lyon, Ecole Centrale de Lyon, LTDS UMR CNRS 5513, 36 Avenue Guy de Collongue, 69134 Ecully, France

${ }^{2}$ Université Paris Est, ICMPE (UMR 7182), CNRS, UPEC, 2 Rue Henry Dunant, 94320 Thiais, France

${ }^{3}$ Université de Lyon, Ecole Nationale d'Ingénieurs de Saint Etienne, LTDS UMR CNRS 5513, 58 rue Jean Parot, 42100 Saint Etienne, France

${ }^{4}$ Mines Saint-Etienne, Université de Lyon, CNRS, UMR 5307 LGF, Centre SMS, F-42023

Saint-Etienne, France

\begin{abstract}
Static recrystallization kinetics, at $300{ }^{\circ} \mathrm{C}$, of a hot rolled commercial purity aluminum alloy AA1050 has been investigated using high temperature nanoindentation. Annealing treatments were achieved inside a nanoindentation device to measure corresponding hot nanohardness versus time. Clear evidences of recrystallization phenomenon were deduced from both hardness decrease and pop-in events on load-displacement curves. The recrystallized fraction for increasing annealing time was compared to conventional post mortem characterizations - i.e. EBSD and Vickers hardness -. A good agreement was found between in situ and post mortem characterization methods. High temperature nanoindentation is proved to be a powerful tool for fast characterization of materials' recrystallization kinetics.
\end{abstract}




\section{Introduction}

Measurement of static recrystallization kinetics (SRX) [1] of metallic materials is of primary importance to optimize annealing treatments subsequent to any manufacturing processes inducing thermomechanical loadings. Recrystallization is based on the birth of new grains inside a microstructure which grow at the expense of former grains. The first step - i.e. incubation step - is mainly viewed as the consequence of high dislocation densities within a set of grains, that later result in nuclei almost-free of dislocations by static recovery. The second step - i.e. grain boundary migration step - is governed by the difference in stored energy i.e. local dislocation density gradients - between nuclei and old grains. Recrystallization is complete when no former grains remain in the microstructure. As a matter of fact, recrystallized materials are significantly softer than as-received materials and can exhibit somewhat different microstructures in terms of texture and anisotropy. For instance, the strong cube texture in recrystallized rolled aluminum sheets results in a plastic anisotropy which can be detrimental to deep drawing [1].

In the framework of classical hot deformation - i.e. forging, rolling, stamping -, investigations on SRX can be conducted macroscopically on bulk samples. For instance, a set of experiments based on isothermal annealing treatments and subsequent hardness characterizations is known to be rich enough to calibrate recrystallization kinetics based on the Johnson-Mehl-Avrami-Kolmogorov (JMAK) equation [1,2]. Refined experimental investigations based on Electron back scattering diffraction (EBSD) have proved their ability to cross the bridge from microstructure features to recrystallization kinetics [1]. Some in situ annealing experiments were even developed to track in real time this phenomenon using neutron diffraction [3], EBSD [4,5] and Synchrotron-based set-up [6,7]. However, such investigations are very time consuming and costly and require careful experimental procedures.

Recent developments in advanced manufacturing processes such as additive manufacturing $[8,9]$ or surface mechanical processing $[10,11]$ led to materials exhibiting high microstructure gradients at a very small scale. Static recrystallization investigation of such heterogeneous microstructures is one of the major issues to address in the coming years, both for academic and industrial needs. It requires to characterize such a phenomenon at a very local scale. 
Nanoindentation testing could be another useful technique for this purpose. Nanoindentation is commonly used to measure hardness, which can be related to the yield strength, and elastic modulus at a submicron-scale [12]. It even allows to map mechanical properties gradient in relation with microstructure $[13,14]$. Moreover, through the analysis of pop-in events on loading curve, which are sudden increases of the indentation depth at a constant applied stress, dislocation density can also be studied [15-17]. That is why nanoindentation is a very promising tool to locally investigate recrystallization phenomena. Up to now, nanoindentation has been used as a post mortem technique to measure recrystallization kinetics [1,18-20]. Thus, in situ characterization seems more suited to the investigation of materials microstructure evolution.

In the recent years, strong efforts have been made to develop in situ high temperature devices for nanoindentation making them more and more efficient regarding thermal stability [21-23]. This paper proposes a new methodology to investigate recrystallization kinetics by using high temperature nanoindentation. Static recrystallizations at $300{ }^{\circ} \mathrm{C}$ were performed on a hot rolled commercial purity aluminum AA1050 up to $3 \mathrm{~h} 30$ annealing time. Annealing treatments were achieved inside a nanoindentation device to measure corresponding hot nanohardness versus time. We show in this paper that high temperature nanoindentation allows for fast and economical recrystallization kinetics characterization.

\section{Material and methods}

The initial material was a hot rolled $1 \mathrm{~mm}$ thick plate of commercial purity aluminum AA1050. This material was chosen because its static recrystallization kinetics has been extensively studied and its recrystallization features are now well known [19,24]. The plate was cut to obtain samples with a volume of $12 \times 10 \times 1 \mathrm{~mm}^{3}$. Samples were prepared using mechanical polishing up to $1 \mu \mathrm{m}$ diamond solution. Each sample was then finished with colloidal silica on a vibrometer during 12 hours. A Vickers grid pattern was performed to locate the characterization zones precisely. Microstructures prior and after in situ nanoindentation annealing were investigated by means of EBSD using a Zeiss Supra 55 VP microscope operating at 20 $\mathrm{kV}$. Maps of the Vickers grid were created with a step size of $600 \mathrm{~nm}$ with an indexation rate 
higher than $95 \%$.

Indentations were performed using a Hysitron TI950 ${ }^{\circledR}$ (Bruker Ltd., Minneapolis, Minnesota) with a specific Nova ${ }^{\circledR}$ temperature stage. Nominal resolutions are $0.02 \mathrm{~nm}$ for displacement and $1 \mathrm{nN}$ for load. An unused Berkovich diamond tip was set up on the apparatus. Tip calibration has been performed at ambient temperature on fused quartz prior to in situ annealing experiments. The error in displacement measurement induced by the tip thermal expansion is mainly the consequence of the non-self-similar tip defect. This is thus more pronounced at very shallow depth. Nevertheless it remains very low compared to other sources of uncertainties like thermal drift or initial tip calibration [25]. Moreover, Wheeler et al. [26] scanned with an atomic force microscope (AFM) a diamond Berkovich tip which underwent long-term usage at temperature up to $400{ }^{\circ} \mathrm{C}$. They did not notice any damage of the tip. Samples were positioned between bottom and top heating plates of the temperature stage. The top plate was clamped with springs to maintain a constant pressure on the sample, even at high temperature. A nitrogen flow was maintained constant at $1.4 \mathrm{~L} / \mathrm{min}$ during the annealing treatment in order to limit the sample oxidization [21]. The tip was heated by convection using the top plate as a micro-oven. Homogeneous temperature state around the indented zone was achieved thanks to the constant nitrogen flow. A clear illustration of the heating stage was given by $\mathrm{Li}$ et al. [27]. The test procedure consisted in indenting the sample at different times once the set temperature was reached (see Fig. 1). A minimum time of 10 min was necessary to lower the displacement drift linked to the temperature stabilization of the tip and the sample. Hence no indentation has been performed for shorter annealing time. Each indentation test took 5 min so a minimum interval of 7 min was achieved between collected data (see Fig. 1).

Tests were performed on two different specimens for which the annealing durations were 165 and 210 min, respectively. Each indentation was performed with a constant strain rate loading of $\frac{\dot{h}}{h}=0.1 s^{-1}$ - i.e. the loading rate increases exponentially with increasing time [28] - up to a maximum load of $7 \mathrm{mN}$ maintained for $5 \mathrm{~s}$ and then unloaded during $5 \mathrm{~s}$ at a constant loading rate. The continuous stiffness measurement (CSM) was set up at a frequency of $200 \mathrm{~Hz}$ and an amplitude varying between 0.4 and $1 \mathrm{~nm}$. Indents were spaced by, at least, $50 \mu \mathrm{m}$ which represents 4 times the size of the final imprint. For each indentation, drift rate was 
evaluated while maintaining a constant load of $2 \mu \mathrm{N}$ prior to the loading procedure. Load was maintained during $1 \mathrm{~min}$ and drift rate was measured from the last $20 \mathrm{~s}$ of the dwell time [27]. Typical drift rates were measured between $-2 n m . s^{-1}$ and $-0.1 n m . s^{-1}$. Penetration depths were corrected from thermal drift considering a linear evolution of displacement since drift rate acquisition was performed on the same time-scale as the loading procedure.

Continuous stiffness measurement has been used to calculate elastic modulus and hardness versus penetration depth by the Loubet's method [29] in order to account for pile-up phenomenon. Presence of the later ones has been assessed by post mortem topographic measurements performed with the Scanning probe microscopy (SPM) module from Hysitron TI950 ${ }^{\circledR}$. Softening during annealing was evaluated and transformed into recrystallized fractions by using the relation [30]:

$$
X_{R E X}=\frac{H_{d e f}-H_{t}}{H_{d e f}-H_{R E X}}
$$

Where $H_{d e f}, H_{R E X}$ and $H_{t}$ are respectively hardness values of the deformed state, the fully recrystallized state and after $t$ minutes at the annealing temperature. Recrystallization kinetics was also investigated using the standard process of multiple isothermal annealing time and post mortem hardness measurements. A set of ten $12 \times 10 \times 1 \mathrm{~mm}^{3}$ samples was thermally treated in a molten salt bath prior to measure their Vickers hardness using a load of $1 \mathrm{kgf}$. Samples were maintained in molten salt at $300{ }^{\circ} \mathrm{C}$ for time ranging from $1 \mathrm{~min}$ to $3 \mathrm{~h}$. After each heating process, samples were water quenched. Samples were then successively polished up to $3 \mu \mathrm{m}$ diamond solution and hardness was measured 5 times on each sample. The given Vickers hardness and the corresponding error bars are respectively the average and standard deviation of those measurements.

\section{Results}

Fig. 2 shows the microstructure of specimen 1, as received and after $2 \mathrm{~h} 45$ annealing at $300{ }^{\circ} \mathrm{C}$ in the nanoindentation set-up. The orientation maps are presented using the inverse pole figure (IPF) color code combined with the distribution of grain boundaries. Bold black lines are high angle boundaries $(\mathrm{HAB})$ - i.e. misorientation $>15^{\circ}-$ and red lines are low 


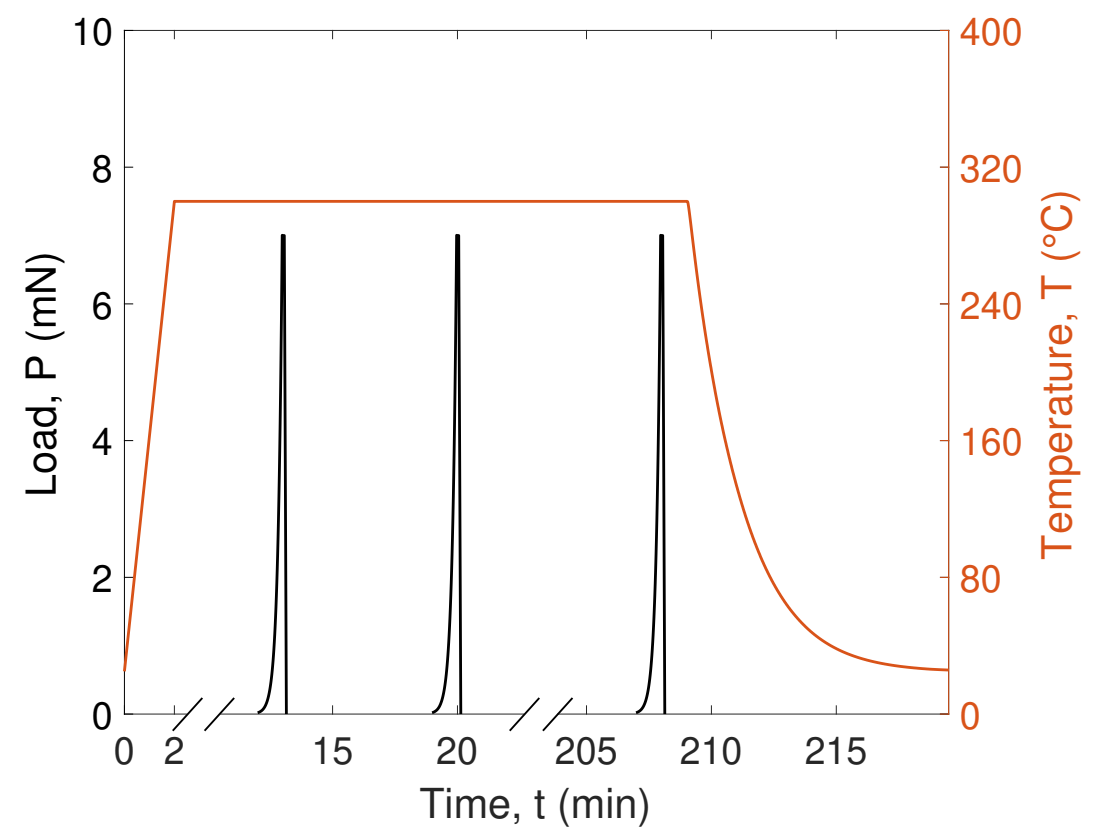

Figure 1: High temperature nanoindentation loading kinematics - samples were heated up rapidly to $300{ }^{\circ} \mathrm{C}$ in the nanondentation set-up. Temperature was kept constant during an holding period of $210 \mathrm{~min}$ (3h30) before rapid cooling. After drift stabilization, nanoindentation measurements were performed in situ every 7 minutes. Black and red curves are indentation and temperature loadings, respectively.

angle boundaries $(\mathrm{LAB})$ - i.e. misorientation $>2^{\circ}$. As expected, the initial material shows a hot rolled microstructure with a few recrystallized grains. It is composed of extruded grains lying parallel to the rolling direction $(\mathrm{RD})$. These grains are subdivided into a network of LAB, revealing the high intragranular stored deformation energy. Consequently, it is more convenient to define a subgrain size, which is close to $1 \mu \mathrm{m}$, than a grain size to analyze nanoindentation results. The crystallographic texture (Fig. 3.a) is close to the one observed by Miszczyk et al. [19] after rolling. After annealing, recrystallization features are clearly visible. Grains are equiaxial, with an average grain size close to $50 \mu \mathrm{m}$, with no intragranular LAB networks inside, except for a few remaining non-recrystallized small grains. The recrystallization is thus almost complete. The crystallographic texture reflects the development of strong cube orientation that is also an evidence of the recrystallization process in such hot rolled materials $[24,31]$ (see Fig. 3.b). Note that blue down-pointing triangles on Fig. 2.d correspond to indent positions. Their size is close to the real imprints to visualize the measurement scale. One indent lies on several micro grains (typically 5 to 10) at the beginning 
(a)
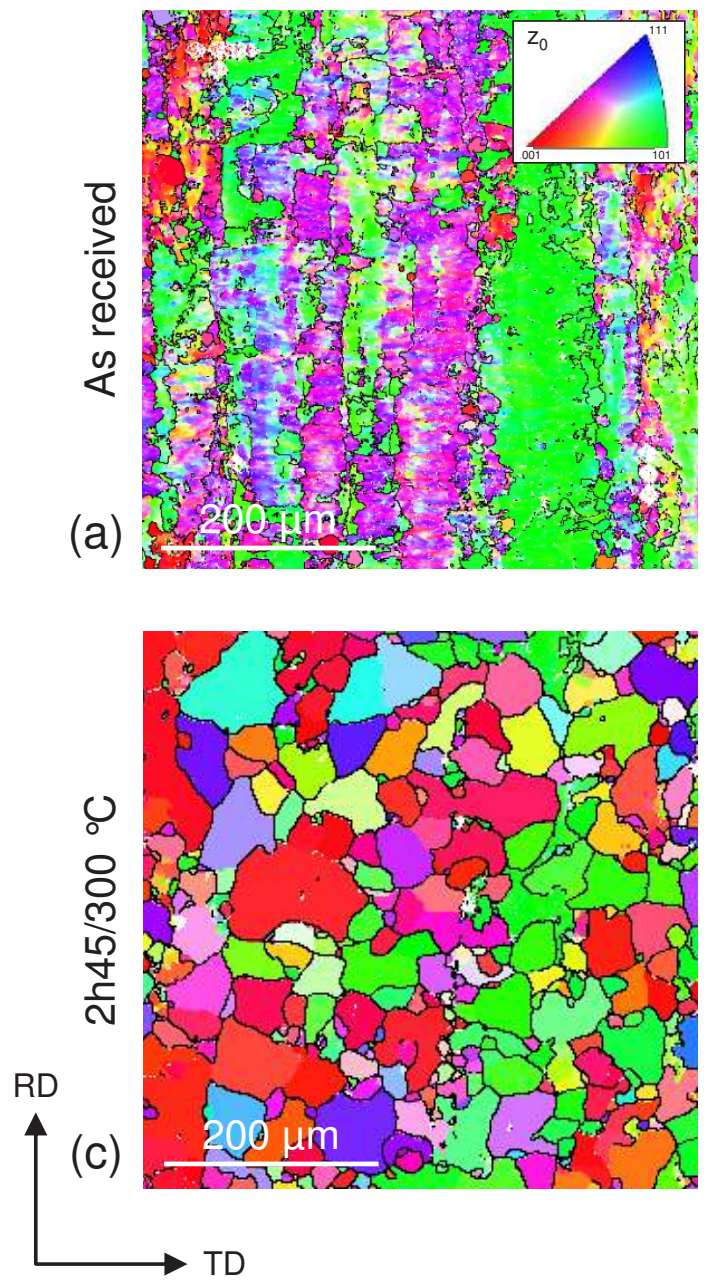

(b)

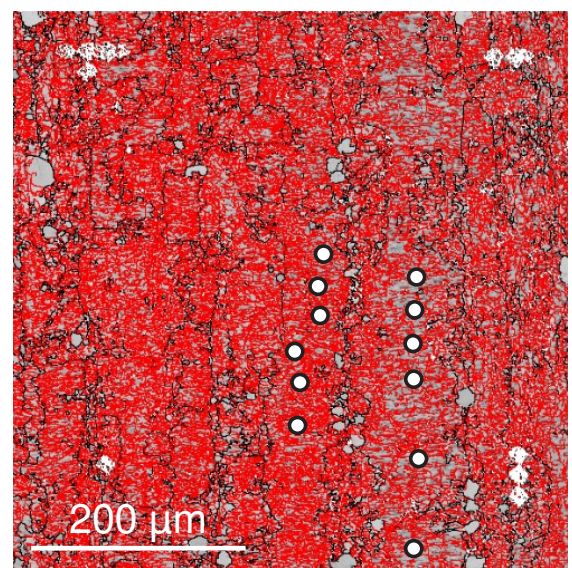

(d)

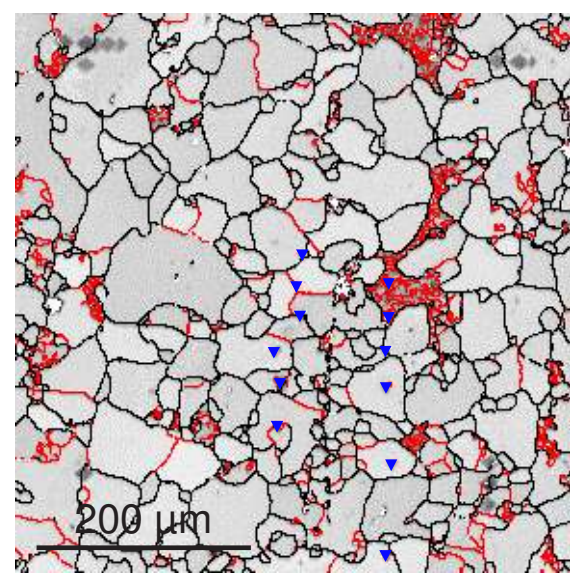

Figure 2: Microstructures of the as received AA1050 (a,b) and annealed during 2h45 at $300{ }^{\circ} \mathrm{C}(\mathrm{c}, \mathrm{d})$ observed at the same location (specimen 1). (a,c) are IPF maps and (b,d) are band contrast maps. Black and red lines represent grain boundary with more than $15^{\circ}$ and $2^{\circ}$ misorientation, respectively. White circles represent the locations of the future indents (b). Blue down pointing triangles highlight the location of the indents performed in situ (d). Maps are oriented along the Rolling/Transverse Directions (RD)/(TD). 
of the annealing process whereas after $2 \mathrm{~h} 45$ annealing the majority of indents lies on a single grain.

Fig. 4 shows load-displacement curves performed on specimen 2 after different annealing durations. It must be noticed that indentation data shown on Fig. 4 and Fig. 6.a are not equally spaced in time, contrary to the experimental procedure. Indeed, some indentation tests failed due to incorrect detection of the contact location and to tip adherence during withdrawal. Load-displacement curves from specimen 1 are similar and exhibit the same trends with increasing annealing time. For clarity, only loading curves are displayed. Color gradient represents the annealing time. The penetration depth increases with increasing annealing time, which reflects the clear softening tendency. Let us note here that there is no correlation between annealing time and drift rate. This points out that the apparent softening trend is not a thermal drift artifact. Fig. 5 displays elastic modulus and hardness versus penetration depth, as measured by CSM, for different annealing times. Presented curves correspond to data displayed on Fig. 4. Elastic modulus measurements are quite noisy. This is a consequence of the low displacement CSM amplitude - i.e. 0.4 to $1 \mathrm{~nm}-$. Nevertheless, mean data calculated between 850 and $900 \mathrm{~nm}$ lay in the range of 55 and $77 \mathrm{GPa}$. Regarding measurement scattering, data remain constant over annealing time whatever the microstructural state, as expected. A typical indentation size effect (ISE) can be seen on the hardness measurement (Fig. 5.b) made at $29 \mathrm{~min}$. It can be related to geometrically necessary dislocations (GNDs) related to plastic flow around the tip [15,32]. For higher annealing times a pop-in event is detected around $50 \mathrm{~nm}$ indentation depth (see detailed view of Fig. 4) and hardness dramatically drops. For every test, hardness can be considered as constant at large indentation depth. Hence, data shown on Fig. 6.a are average values determined over a constant range of 850 to $900 \mathrm{~nm}$. Results from the post mortem Vickers indentations which were performed after multiple isothermal annealing durations are compared to the later ones on Fig. 6.a. The axis of Vickers hardness - i.e. right axis - was scaled compared to the nanohardness - i.e. left axis - so as to highlight a similar hardness drop. Indeed, Vickers hardnesses show a drop of $20 \%$ after $3 \mathrm{~h}$ annealing at $300{ }^{\circ} \mathrm{C}$ while in situ nanoindentation hardnesses show a loss of $50 \%$ after the same annealing duration. Fig. 6.b displays the maximum pop-in amplitude for each indentation. One can see two specific regimes - one without pop-in and 


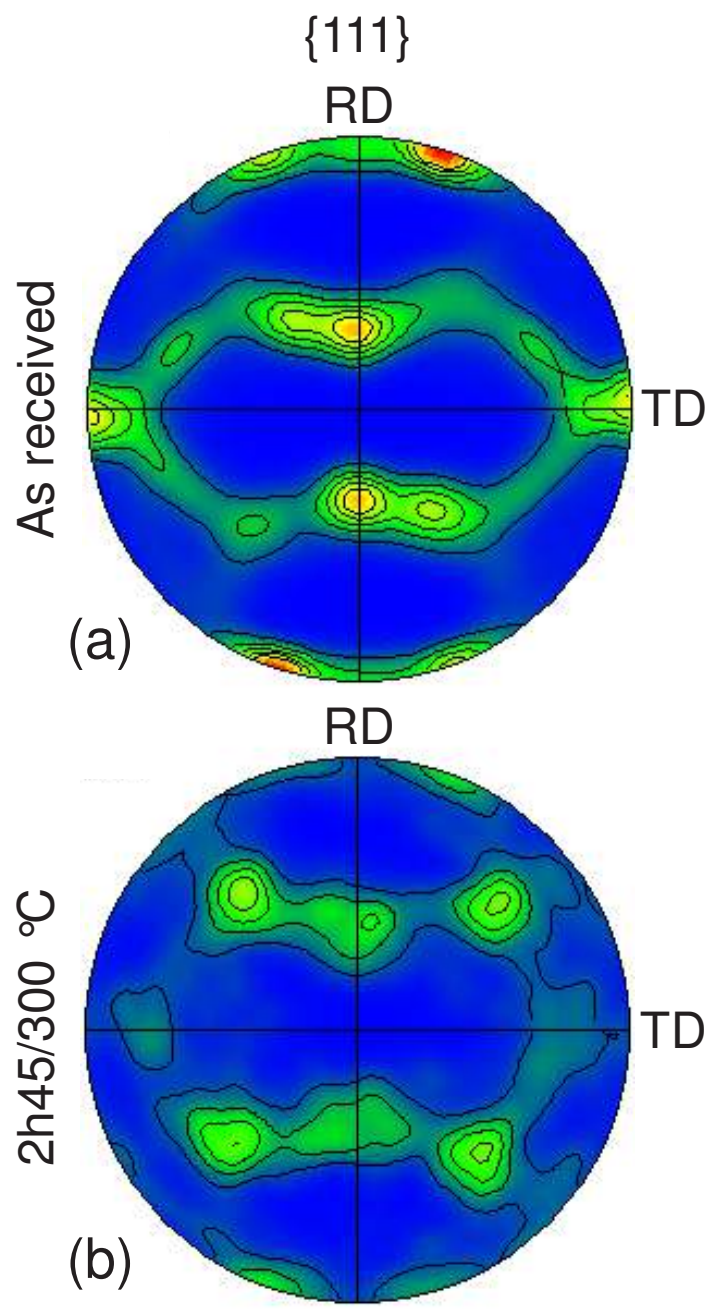

Figure 3: Textures of as-received AA1050 (a) and annealed for $2 \mathrm{~h} 45$ at $300{ }^{\circ} \mathrm{C}$ (b). Pole figures correspond to the EBSD maps presented in Fig. 2.

one with pop-in exceeding $20 \mathrm{~nm}$. The transition between these two regimes is closely related to the hardness decrease. Even so, no direct relation can be established between hardness and pop-in amplitude.

\section{Discussion}

\subsection{Evidence of recrystallization phenomenon on load-displacement curves}

Recrystallization and recovery phenomena lead to dislocation density decrease since the amount of new undeformed grains increases [1]. In the meantime, hardness is closely related to 


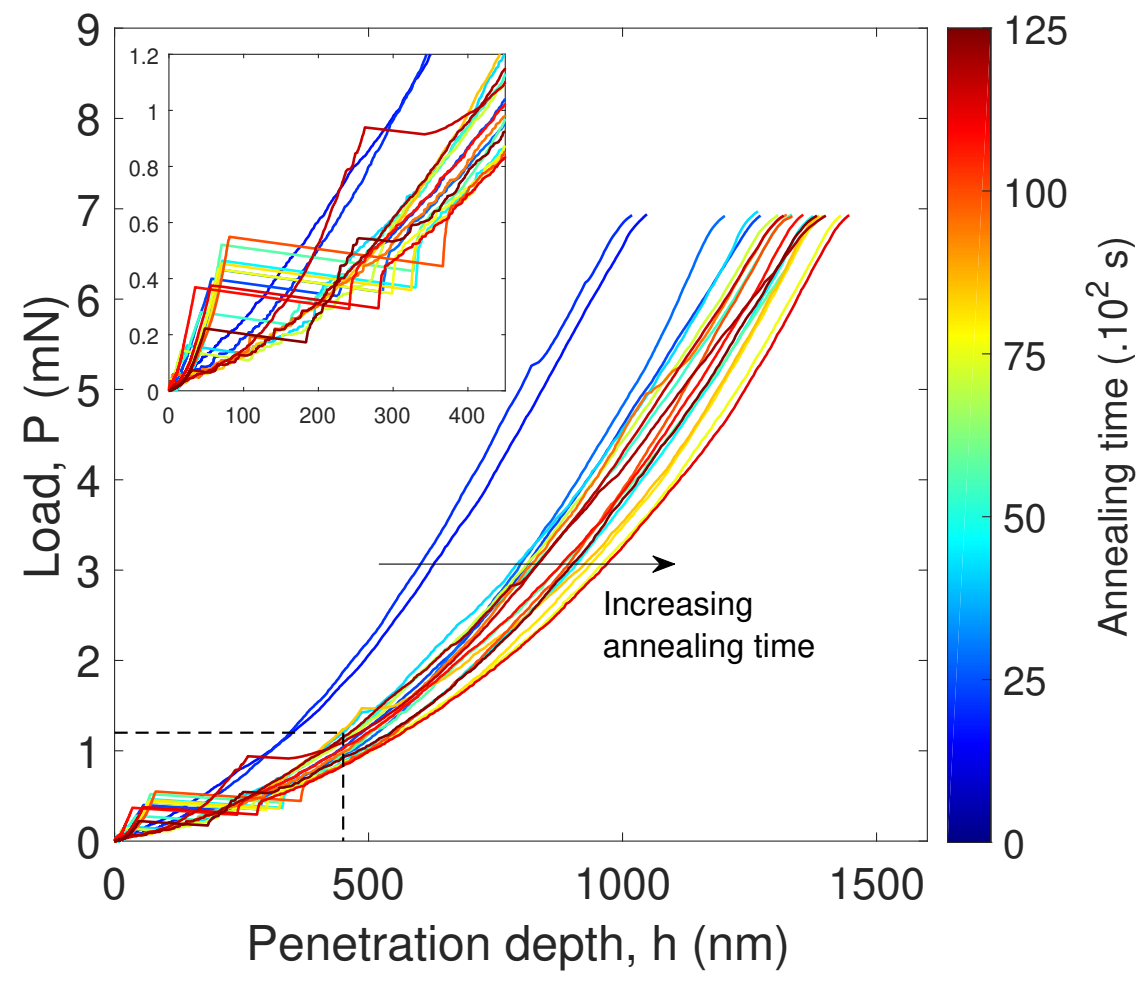

Figure 4: Load displacement curves for a set of in situ nanoindentation measurements (specimen 2). For clarity, only loading curves have been displayed. The color scale is related to the time for which one indentation was performed after heating up to $300{ }^{\circ} \mathrm{C}$. A detailed view of low load mechanisms is given. Two distinct regimes are observed : one with pop-in and an other without. 

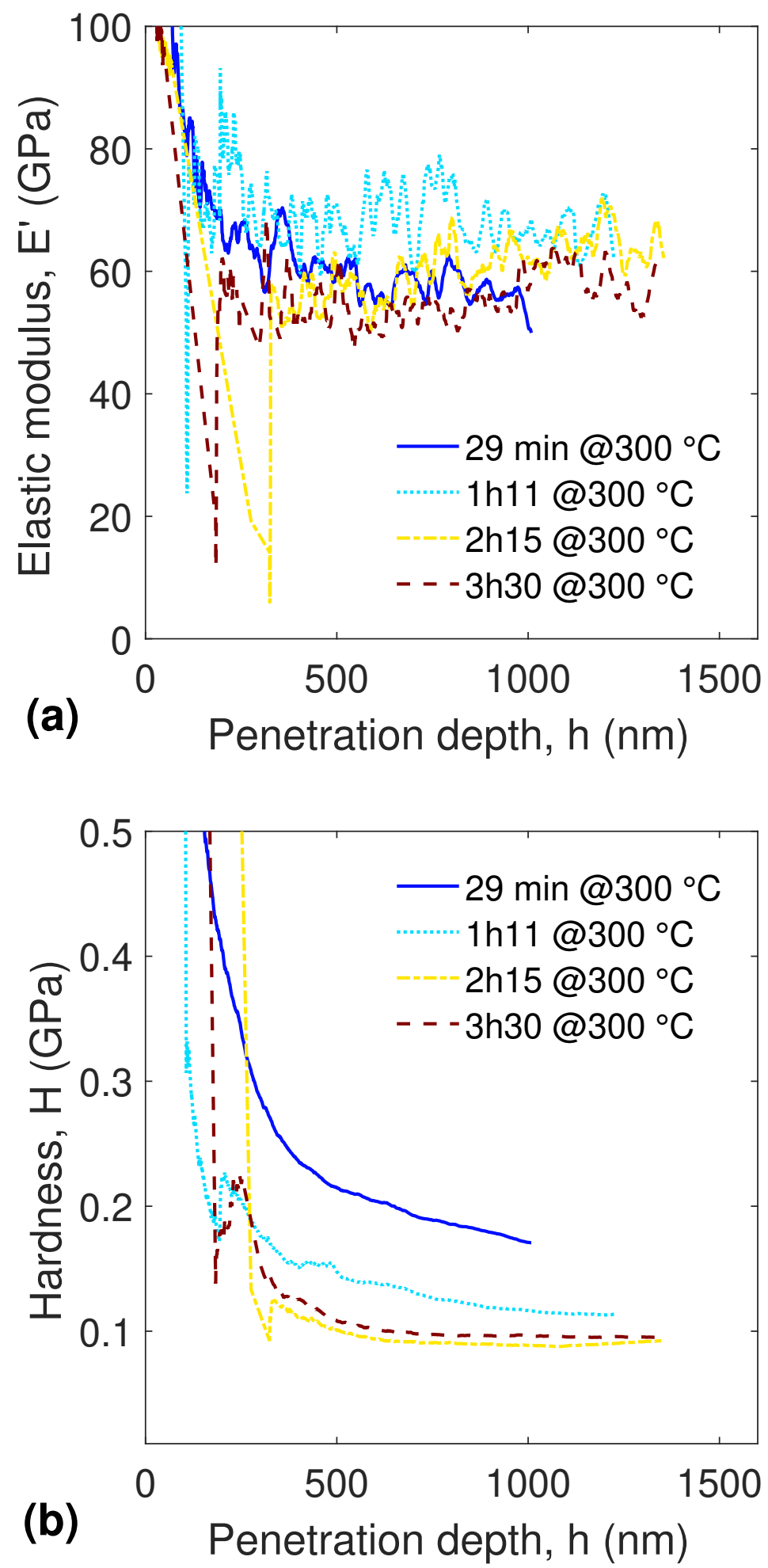

Figure 5: Elastic modulus vs. penetration depth (a) and hardness vs. penetration depth (b) measured from continuous stiffness measurement during a set of in situ nanoindentation (specimen 2). 

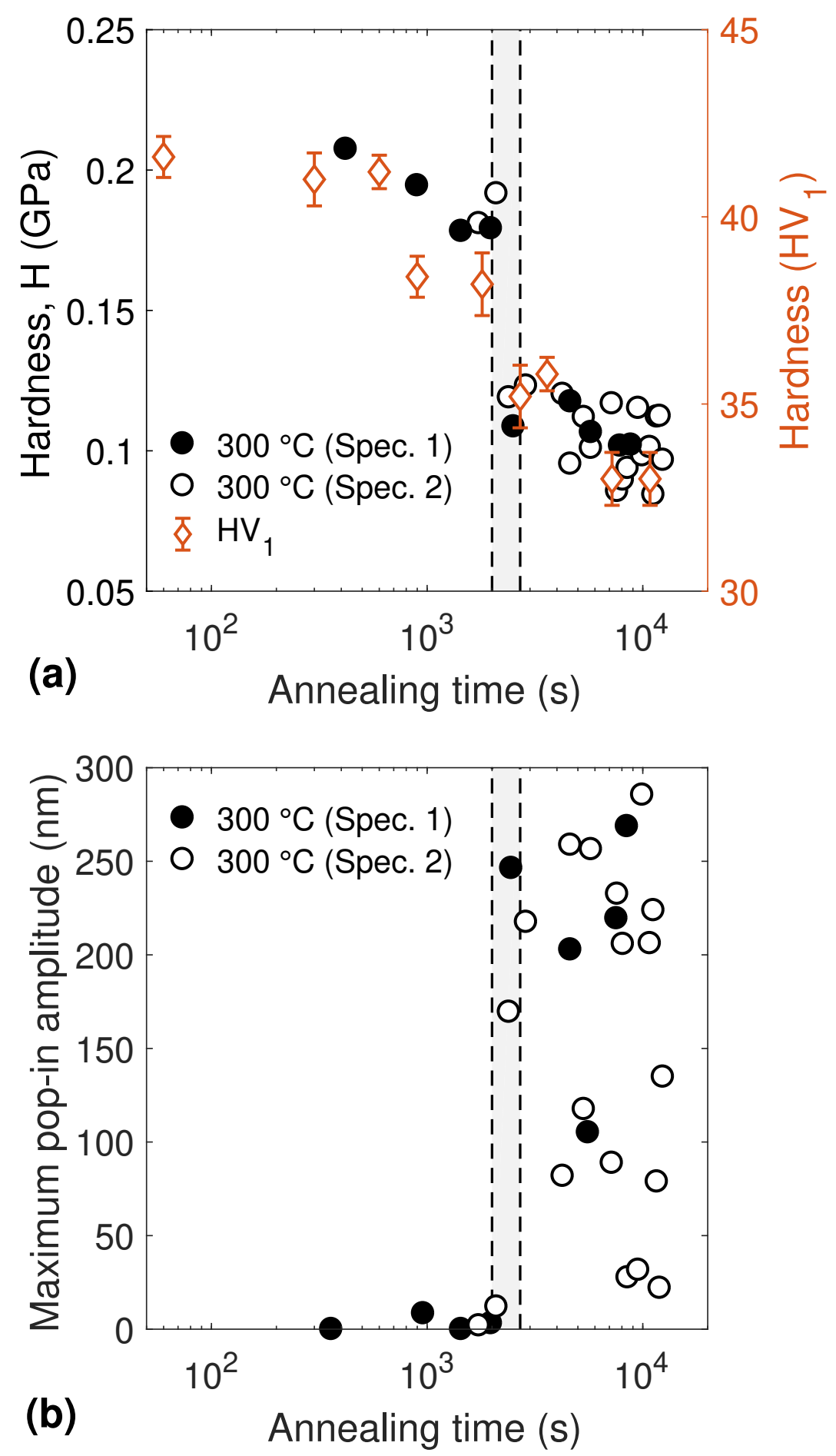

Figure 6: Mean nanoindentation hardness calculated between 850 and $900 \mathrm{~nm}$ from CSM measurements at $300{ }^{\circ} \mathrm{C}$ and Vickers hardness measured at different annealing times (a). Maximum pop-in amplitude measured by nanoindentation in situ at different annealing times (b). The light gray band highlights the transition range of pop-in events, closely related to the hardness drop. 
yield stress [33] which depends on dislocation density. The model proposed by Nix \& Gao [32] and modified by Durst et al. [15] expresses the hardness as a function of statistically-stored and geometrically necessary dislocations (respectively, SSDs and GNDs). In this model SSDs density is related to the material's initial state and GNDs density is proportional to the inverse indentation depth. At large indentation depth, the effect of GNDs density is negligible making hardness measurements mainly rely on SSDs density. Thus, change in hardness is closely associated with SSDs density variation. It has been shown on Fig. 6.a that hardness decreases according to annealing time. Hence, this is a clear signature of the recrystallization phenomenon. Regarding hardness versus penetration depth, one can expect a large ISE with the initial microstructure state due to near-surface hardening and microstructure heterogeneities. ISE should then be reduced during the recovery stage. Still, it would need a larger set of indentation tests as well as a deeper microstructural investigation to consider this point. Let us note that the analysis of indentation size effect in recrystallized grains is quite difficult because of pop-in events which significantly disturb hardness measurements.

Another confirmation of the recrystallization phenomenon is given by the study of pop-in. On Fig. 6.b, pop-ins appear only after a certain annealing time, around 35 min. Barnoush [16] showed that the presence of pop-in on aluminum alloys is driven by dislocation density rather than by breakage of alumina oxide layers. This can be explained by the probability to trigger dislocation sources underneath the tip - i.e. the greater the dislocation density, the lower the probability to generate pop-in -. Montagne et al. [17] confirmed that the presence of pre-existing dislocations promotes the nucleation of new dislocations and hence, lowers the load necessary to generate pop-in. As a result, apparition of large pop-in can be related to SSD density reduction and therefore associated to recovery and recrystallization phenomena. It is interesting to note that the transition from small to large pop-in amplitude events, highlighted by the light gray band on Fig. 6.b corresponds to the hardness transition. From these observations, it is clear that the recrystallization rate attains its maximum at around $35 \min (2100 \mathrm{~s})$. 


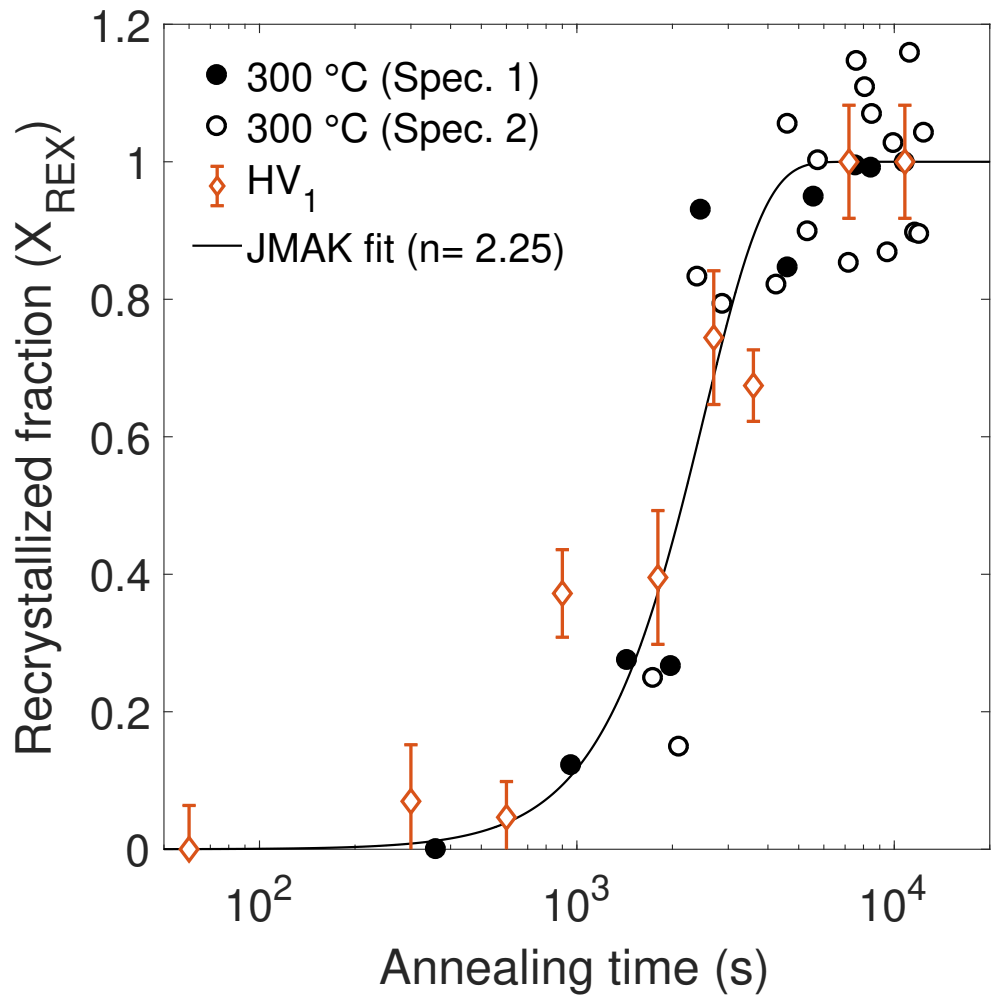

Figure 7: Recrystallization kinetics deduced from nanoindentation hardness and Vickers hardness. Black curve represents the JMAK equation fit over the nanoindentation hardness data. 


\subsection{Recrystallization kinetics}

Fig. 7 shows the recrystallization kinetics obtained from in situ nanohardness and post mortem Vickers measurements - i.e. conventional method -, as calculated from Eq. (1). Recrystallized fraction deduced from nanohardness has been calculated taking into account the maximum hardness of the two sets of data as the initial state $\left(H_{d e f}\right)$, and the average hardness after $2 \mathrm{~h}$ annealing as the fully recrystallized state $\left(H_{R E X}\right)$ (see Fig. 6.a). A very good agreement is observed despite the slightly different heating procedure between in situ and post mortem characterizations. Let us note that Alvi et al. [24] measured a recrystallization time - i.e.time to achieve $50 \%$ of recrystallization - close to $40 \mathrm{~min}(2400 \mathrm{~s})$ at $325{ }^{\circ} \mathrm{C}$ on the same alloy. This is consistent with our results regarding differences in rolling conditions and annealing temperature.

Time evolution of the recrystallized fraction, $X_{R E X}$, can be modeled by the JMAK theory $[1,2]:$

$$
X_{R E X}=1-\exp \left(-B t^{n}\right)
$$

With $B$, a constant and $n$, the Avrami exponent. Data of samples 1 and 2 were successfully fitted using Eq. (2). No incubation time has been taken into account to fit this JMAK equation. The recrystallization time at $300{ }^{\circ} \mathrm{C}$ extracted from the JMAK fitting is $35 \mathrm{~min}$ which corresponds exactly to the experimentally observed transition after $35 \mathrm{~min}$. A value of 2.25 was found for the exponent $n$. This may point out that the recrystallization kinetics would be driven by a site saturated growth in 2-D [1], which would be consistent with the large 2-D deformation induced by the rolling process. However, it has to be considered that there are already some recrystallized grains present in the very beginning. Moreover the plastic zone around an indent is only a few micron deep. It is akin to several subgrains in the non-recrystallized state but becomes representative of only one grain in the recrystallized state. Hence, recrystallization kinetics extracted from nanoindentation experiments are more likely to be affected by the grains' free-surface, especially when indentations are performed on recrystallized grains. Regarding this concern, the Avrami exponent deduced from this study has to be interpreted with care. Such analysis would require an extensive set of data to obtain 
a representative value of this exponent.

\subsection{Advantages and opened questions}

In situ annealing nanoindentation was proposed as a new technique to investigate recrystallization kinetics. Compared to post mortem techniques, in situ annealing nanoindentation is time, sample and cost-saving. Indeed, this technique allows the investigation of recrystallization on a single sample in a limited amount of time - i.e. the chosen annealing duration -. Hence it opens the way to screen the effects of various parameters on recrystallization, such as alloy composition, pre-treatments, surface treatments, in an efficient way compared to conventional post mortem methods. Moreover, this local mechanical technique should allow the study of recrystallization phenomena of thin films or of each phase of multi-phased materials for example. Compared to other in situ techniques, such as EBSD or synchrotron based set-up, in situ annealing nanoindentation appears as a simpler equipment, which should be easier to use and less expensive. Even so, all those in situ techniques have to be considered as complementary since they are able to measure different features of the same phenomenon.

We shall now consider some questions which arise from this work. The implicit small scale of nanoindentation testing regarding the heterogeneous as-received microstructure and microstructure evolution happening during recrystallization is discussed. Indeed, at the micronscale, nuclei are already present in the as-received material (see Fig. 2.b). Local recrystallization kinetics is thus very dependent on the location of these nuclei. In the same way, non-recrystallized regions can be clearly evidenced despite the fact that recrystallization appeared almost complete macroscopically (see Fig. 2.d). As a result, measurements could be strongly affected by the indentation location and it would require statistically representative data to conclude on the average recrystallized fraction at macroscopic scale. Our study does not present a large enough number of collected data to obtain such an average. However a clear trend can be extracted from the two set of experiments performed in situ. The scattering from hardness measurements after $2 \mathrm{~h}$ at $300{ }^{\circ} \mathrm{C}$ is about $32 \%$ around the mean value. Considering the assumption of a non-homogeneous recrystallization, this is quite low, as we would expect hardness varying between maximum - i.e. non-recrystallized - and minimum - i.e. recrystallized - even after long annealing time. It could be explained by the relatively small 
probed area (around $300 \times 300 \mu \mathrm{m}$ ) and the care taken to locate the indents at the center of the extruded non-recrystallized grains (see Fig. 2.b), implying a similar initial microstructure. Current developments of ultra-fast nanoindentation testing at high temperature should help to address this issue. It might open the way to measure macroscopic recrystallization kinetics in relation to heterogeneities of microstructural changes at very small scale.

Another significant issue to address is the indentation apparatus thermal stability and especially its critical stabilization time. It has been highlighted in section 2 that a time of $10 \mathrm{~min}$ after annealing starts was necessary to lower the drift rate related to isothermal stabilization of the contact zone. Even though, drift rates remain not negligible whatever the annealing time and have to be considered in the data post-processing. Moreover, the loading procedure itself is performed in a given duration which has to be negligible compared to the recrystallization kinetics. In this study the loading segment is achieved in $1 \mathrm{~min}$ and the period between each indentation is of $7 \mathrm{~min}$ at least. Therefore, fast recrystallization process - i.e with a 50\%-recrystallized time under $20 \mathrm{~min}$ - or incubation time cannot be characterized. As already expressed, ultra-fast nanoindentation would allow to access shorter recrystallization phenomena with a better time resolution. Yet, mechanical properties of metals at relatively high temperatures are known to strongly depend on indentation strain rate (ISR). Hardness would be expected to increase with increasing ISR. This should not substantially modify the measured recrystallization kinetics since the method relies on relative hardness changes.

To the same extent, it has been assessed by several authors that recrystallization is affected by plastic deformation zone $[34,35]$. Humphreys et al. studied the recrystallization phenomenon of deformed aluminum at the vicinity of undeformed particles [34]. He explains that higher SSDs and GNDs concentration, due to plastic deformation around undeformed particles, promotes nucleation and thus recrystallization. In a similar manner, the tip penetration in the material generates GNDs which extend the content of already present dislocations [15]. Nanoindentation could enhance nucleation and hence modify the local recrystallization kinetics, especially for short annealing time for which recrystallization is mainly driven by nucleation rate. Nevertheless, potential effects seem limited in the light of the good correlation obtained with post mortem hardness measurements. 


\section{Conclusion}

A new characterization method of recrystallization kinetics based on in situ annealing treatment in a nanoindentation device has been developed. Recrystallization process is evaluated from high temperature nanohardness measurements. Microstructure and texture observations have been performed before and after isothermal annealing at $300{ }^{\circ} \mathrm{C}$. Conventional hardness method has been used to extract recrystallization kinetics and to compare to in situ results. The main inputs from this study are listed below :

- Static recrystallization was clearly evidenced through the decrease of hardness with annealing time and by the sudden apparition of pop-in in the load-displacement curves.

- The proposed methodology gives very consistent results compared to EBSD microstructural investigations performed on the same material by Alvi et al. [24] and post mortem hardness measurements.

- Recrystallization kinetics can be investigated from a single specimen and within a limited experiment time, contrary to conventional techniques - i.e. post mortem hardness and metallography-.

In future work, indentation size effect evolution with annealing time should be considered more precisely. This would allow for a deeper understanding of the SSD distribution variation during annealing. Furthermore, the intrinsic thermomechanical loading that is induced by nanoindentation at elevated temperature might play a role on recrystallization and thus deserves to be addressed.

For now, hot nanoindentation experiments are relevant for the characterization of relatively slow recrystallization kinetics because of their inherent thermal stabilization time and loading procedure duration. In the future, ultra-fast nanoindentation should open the way to characterization of shorter recrystallization time and of macroscopic recrystallization kinetics in relation to local heterogeneities of microstructural changes. 


\section{Acknowledgements}

This work was supported by the LABEX MANUTECH-SISE (ANR-10-LABX-0075) of Université de Lyon, within the program "Investissements d'Avenir" (ANR-11-IDEX-0007) operated by the French National Research Agency (ANR). The authors would also like to acknowledge financial support from Institut Carnot Ingénierie@Lyon. G. Blanc and M. Mondon from EMSE are thanked for their kind support on sample preparation and EBSD measurements. Finally, authors acknowledge I. Guillot and G. Bracq from ICMPE for fruitful discussions.

\section{Data availability statement:}

The raw/processed data required to reproduce these findings cannot be shared at this time due to technical or time limitations. 


\section{References}

[1] F. Humphreys and M. Hatherly. Recrystallization and Related Annealing Phenomena: Second Edition. Elsevier, 2004.

[2] R.D. Doherty, D.A. Hughes, F.J. Humphreys, J.J. Jonas, D.Juul Jensen, M.E. Kassner, W.E. King, T.R. McNelley, H.J. McQueen, and A.D. Rollett. Current issues in recrystallization: a review. Materials Science and Engineering: A, 238(2):219-274, 1997.

[3] F. Christien, M. T. F. Telling, K. S. Knight, and R. Le Gall. A method for the monitoring of metal recrystallization based on the in-situ measurement of the elastic energy release using neutron diffraction. Review of Scientific Instruments, 86(5):053901, 2015.

[4] A. Lens, C. Maurice, and J. H. Driver. Grain boundary mobilities during recrystallization of Al-Mn alloys as measured by in situ annealing experiments. Materials Science and Engineering A, 403(1-2):144-153, 2005.

[5] N. Bozzolo, S. Jacomet, and R. E. Logé. Fast in-situ annealing stage coupled with EBSD: A suitable tool to observe quick recrystallization mechanisms. Materials Characterization, 70(Supplement C):28-32, 2012.

[6] E. M. Lauridsen, H. F. Poulsen, S. F. Nielsen, and D. Juul Jensen. Recrystallization kinetics of individual bulk grains in 90\% cold-rolled aluminium. Acta Materialia, 51(15):4423$4435,2003$.

[7] E. M. Lauridsen, S. Schmidt, S. F. Nielsen, L. Margulies, H. F. Poulsen, and D. Juul Jensen. Non-destructive characterization of recrystallization kinetics using threedimensional X-ray diffraction microscopy. Scripta Materialia, 55(1 SPEC. ISS.):51-56, 2006.

[8] B. Vrancken, L. Thijs, J.-P. Kruth, and J. Van Humbeeck. Heat treatment of Ti6Al4V produced by Selective Laser Melting: Microstructure and mechanical properties. Journal of Alloys and Compounds, 541:177-185, 2012. 
[9] L. Thijs, K. Kempen, J.-P. Kruth, and J. Van Humbeeck. Fine-structured aluminium products with controllable texture by selective laser melting of pre-alloyed AlSi10Mg powder. Acta Materialia, 61(5):1809-1819, 2013.

[10] G. Kermouche, G. Jacquet, C. Courbon, J. Rech, Y.Y. Zhang, and R. Chromik. Microstructure Evolution Induced by Sliding-Based Surface Thermomechanical Treatments - Application to Pure Copper. Materials Science Forum, 879:915-920, 2016.

[11] D. Tumbajoy-Spinel, X. Maeder, G. Guillonneau, S. Sao-Joao, S. Descartes, J.-M. Bergheau, C. Langlade, J. Michler, and G. Kermouche. Microstructural and micromechanical investigations of surface strengthening mechanisms induced by repeated impacts on pure iron. Materials \&3 Design, 147:56-64, 2018.

[12] W. C. Oliver and G. M. Pharr. An improved technique for determining hardness and elastic modulus using load and displacement sensing indentation experiments, 1992.

[13] D. Tumbajoy-Spinel, S. Descartes, J.-M. Bergheau, V. Lacaille, G. Guillonneau, J. Michler, and G. Kermouche. Assessment of mechanical property gradients after impact-based surface treatment: application to pure $\alpha$-iron. Materials Science and Engineering: $A$, 667:189-198, 2016.

[14] Y. Zayachuk, D. E.J. Armstrong, K. Bystrov, S. Van Boxel, T. Morgan, and S. G. Roberts. Nanoindentation study of the combined effects of crystallography, heat treatment and exposure to high-flux deuterium plasma in tungsten. Journal of Nuclear Materials, 486:183-190, 2017.

[15] K. Durst, B. Backes, O. Franke, and M. Göken. Indentation size effect in metallic materials: Modeling strength from pop-in to macroscopic hardness using geometrically necessary dislocations. Acta Materialia, 54(9):2547-2555, 2006.

[16] A. Barnoush. Correlation between dislocation density and nanomechanical response during nanoindentation. Acta Materialia, 60(3):1268-1277, 2012. 
[17] A. Montagne, V. Audurier, and C. Tromas. Influence of pre-existing dislocations on the pop-in phenomenon during nanoindentation in MgO. Acta Materialia, 61(13):4778-4786, 2013.

[18] L. H. Liao, H. Jin, M. Gallerneault, and S. Esmaeili. Through-thickness recrystallization characteristics of a laminated AA3xxx-AA6xxx aluminum alloy system. Materials Characterization, 101:71-77, 2015.

[19] M. M. Miszczyk, H. Paul, J. H. Driver, and J. Poplewska. The influence of deformation texture on nucleation and growth of cube grains during primary recrystallization of AA1050 alloy. Acta Materialia, 129:378-387, 2017.

[20] Z. Ren, G. Hu, R. Ma, J. Wu, Z. Wang, and C. Luo. Recrystallization and grain growth of U-5.5 wt\%Nb alloy. Journal of Nuclear Materials, 494:284-293, oct 2017.

[21] J. M. Wheeler, D. E. J. Armstrong, W. Heinz, and R. Schwaiger. High temperature nanoindentation: The state of the art and future challenges. Current Opinion in Solid State and Materials Science, 19(6):354-366, 2015.

[22] P. Sudharshan Phani and W. C. Oliver. A direct comparison of high temperature nanoindentation creep and uniaxial creep measurements for commercial purity aluminum. Acta Materialia, 111:31-38, 2016.

[23] J. S.K.L. Gibson, S. Schröders, C. Zehnder, and S. Korte-Kerzel. On extracting mechanical properties from nanoindentation at temperatures up to $1000 \hat{A} \breve{r} \mathrm{C}$. Extreme Mechanics Letters, 17:43-49, 2017.

[24] M. H. Alvi, S. W. Cheong, J. P. Suni, H. Weiland, and A. D. Rollett. Cube texture in hotrolled aluminum alloy 1050 (AA1050)-nucleation and growth behavior. Acta Materialia, 56(13):3098-3108, 2008.

[25] C. A. Schuh, C. E. Packard, and A. C. Lund. Nanoindentation and contact-mode imaging at high temperatures. Journal of Materials Research, 21(03):725-736, 2006. 
[26] J. M. Wheeler, R. A. Oliver, and T. W. Clyne. AFM observation of diamond indenters after oxidation at elevated temperatures. Diamond and Related Materials, 19(11):1348$1353,2010$.

[27] Y. Li, X. Fang, S. Lu, Q. Yu, G. Hou, and X. Feng. Effects of creep and oxidation on reduced modulus in high-temperature nanoindentation. Materials Science and Engineering: A, 678:65-71, 2016.

[28] B. N. Lucas, W. C. Oliver, G. M. Pharr, and J.-L. Loubet. Time Dependent Deformation During Indentation Testing. MRS Proceedings, 436(1):233-238, 1997.

[29] G. Guillonneau, G. Kermouche, J.-M. Bergheau, and J.-L. Loubet. A new method to determine the true projected contact area using nanoindentation testing. Comptes Rendus Mécanique, 343(7-8):410-418, 2015.

[30] R. A. Vandermeer and N. Hansen. Recovery kinetics of nanostructured aluminum: Model and experiment. Acta Materialia, 56(19):5719-5727, 2008.

[31] J. Hirsch, E. Nes, and K. Lücke. Rolling and recrystallization textures in directionally solidified aluminium. Acta Metallurgica, 35(2):427-438, 1987.

[32] W. D. Nix and H. J. Gao. Indentation size effects in crystalline materials: A law for strain gradient plasticity. Journal of the Mechanics and Physics of Solids, 46(3):411-425, 1998.

[33] D. Tabor. The hardness of solids. Review of Physics in Technology, 1(3):145, 1970.

[34] F. J. Humphreys. The nucleation of recrystallization at second phase particles in deformed aluminium. Acta Metallurgica, 25(11):1323-1344, 1977.

[35] R. A. Vandermeer and D. Juul Jensen. Recrystallization in hot vs cold deformed commercial aluminum: A microstructure path comparison. Acta Materialia, 51(10):3005-3018, 2003. 